\title{
A systematic review of the protective and risk factors influenc- ing the mental health of forced migrants: Implications for sus- tainable intercultural mental health practice
}

\author{
Hadi Farahani 1,*, Natalie Joubert, Janet Carter Anand, Timo Toikko and Mohamed Tavakol 2 \\ 1 Department of Social Sciences, University of Eastern Finland, 70211 Kuopio, Finland; njoubert@uef.fi; ja- \\ net.anand@uef.fi (J.C.A.); timo.toikko@uef.fi \\ 2 Department of Social Sciences, University of Tehran, 1411713118 Tehran, Iran; mtavakol@ut.ac.ir \\ * Correspondence: hadif@uef.fi
}

\begin{abstract}
This systematic review followed the guidelines of the Preferred Reporting Items for Systematic Reviews and Meta-Analysis (PRISMA) statement to synthesizes the existing literature with a goal to review current conceptual frameworks of mental health of forced migrants for further practice research priorities in social work. The search was done between January 2015 to January 2021. As a result, 29 studies met inclusion criteria. Medicalizing mental health issues by relying solely on the effectiveness of medicine was a controversial risk factor that negatively affected daily life activities of refugees and reduced their willingness for seeking professional mental health services. Empowering vulnerable minorities by giving them back their power and agency to be able to speak for themselves and raise voices of trauma and recovery was the missing protective factor for a sustainable mental health practice. The benefits of group-based interventions are highlighted in which communities and individuals address mental health issues as well as isolation through building collective identities and support networks. Information and communication technologies (ICTs) can add more strength to any kind of mental health interventions. Finally, benefits of applying ecological perspective for the mental health of refugees, and its implications for a sustainable intercultural practice are discussed. Social workers in this model are the representatives of at-risk groups, thus need more agency and creativity in reflecting client's concrete needs.
\end{abstract}

Keywords: mental health; risk factor; protective factor; refugee; asylum-seeker; sustainable intervention

\section{Introduction}

It is established that exposure to the traumatic experiences associated with forced migration endangers the overall mental health of refugees creating a worldwide mental health crisis [1,2]. The United Nations High Commissioner for Refugees estimates that global forced displacement has surpassed 80 million at mid-2020 including 26.3 million refugees, and 4.2 million asylum seekers [3]. UNHCR defines refugees as people who have been forced to flee their country because of persecution, war or violence, have a wellfounded fear of persecution for reasons of race, religion, nationality, political opinions or membership in a particular social group in the country of their nationality [4]. Whereas asylum-seekers live in a more precarious situation as they must await formal recognition as 'refugee' to be eligible for the protections afforded to those with refugee status [5].

Across Europe and internationally there is a pressing need for the development of culturally appropriate mental health services for socially excluded and marginalized populations [6]. Approaches to mental healthcare are undergoing significant reforms around 
mental health intervention and immigrants' health policies when social scientists realized that mental health and well-being are influenced by various social determinants [5,7]. Through efforts to acquire holistic approaches towards mental health, social scientists admitted that social, cultural, and historical diversity of refugees adds to the complexity of mental health service delivery but also presents opportunities for reform [8,9].

Considering all issues faced by forced migrants, especially in a time that services are stretched thin, social workers play important role in the mental health service delivery for refugee populations, and as a single medium between common top-down policies and forced migrants. Top-down policies place pressure on both social workers and forcibly displaced immigrants [10]. It is imperative that social workers are informed by research about what culturally appropriate interventions can be attempted and which would be most effective. This is not easy in a culturally diverse refugee population with social workers from the host country often very limited in knowledge of refugees mental and cultural landscape. However, the base-line is that it is critical for social workers to understand the mental health needs of this often highly traumatized population as well as having the cultural sensitivity necessary to be successful in helping refugees sustainably integrate into society and avoid mental health pitfalls.

At present, most of the research in the field of migration is reproduced through the same dominant top-down patterns, confined to pathology, and highlighting only prevalence rates of the mental health issues. Thus, voices, interests and expectations of the immigrant communities are ignored. However, with the continued migration of refugees, there is increased attention to how to address the ongoing needs of refugees and has resulted in greater demands for services appropriate to their needs.

Mental health services are one of the most important services that forced migrants urgently need upon arrival to the host countries. However, Watters criticizes the Western Mental Health Care approach in the refugee situation as often coming from a premise which because of trauma and cultural norms may be quite inappropriate [11]. The importance of post-migration stressors to refugee's mental health status suggests the need for sustainable therapeutic interventions with psychosocial elements that address the specific ecology of being a refugee as well as the conditions of refugees' lives. To do so, sustainable therapeutic interventions should fully cognizant of the wide range of cultural diversities in refugees' population, with a predictable dissonance between their concepts of mental health and those of the host country.

\subsection{Literature Review}

Literature shows that mental health of forced migrants has been extensively studied, however studies produced additional puzzles and noncomprehensive frameworks for analysis, and intervention. Research clearly indicates that refugees' mental health is highly influenced by the conditions that they find themselves in post migration [12], often abject squalor such as the camps on the Turco-Syrian border. The clash of cultures, the fear of deportation and the almost ubiquitous instability are only some issues. It is of great concern that refugees who have lived in a host country for more than five years continue, despite this time in a relatively safer environment, show higher rates of depressive and anxiety disorders than the host population [13-17]. It is therefore clear that the stimuli for mental instability continue to have repercussions, whether in post-traumatic stress with nightmare or simply replay of traumas past. Of great concern is a Swedish study which showed higher incidence for psychotic disorders (i.e., more newly diagnosed psychotic disorders) in refugee compared with the host population [18]. This raises the question of epigenetics as an underlying element in people who have been exposed to high levels of trauma over long periods [19]. Recently this recognition has embraced new directions of study for this condition, in particular defining populations with resilience and populations who are prone to PTSD, opening the possibilities of targeted novel PTSD therapies [19].

Other studies on utilizing mental health services by refugees show that despite higher prevalence rates of mental health issues being documented, there still exists an 
underutilization of Western mental health treatment by refugee populations, the reasons given including both structural and cultural barriers [20-22]. Thus existing research on mental health service barriers has identified issues of stigma, distrust of services and social and cultural problems that impact on "how problems are understood" and the question about whether help should be sought and if so, how" [23-25]. It is therefore important for social workers to understand the barriers and reasons why underutilization exists and how to better support this vulnerable population [21,26].

\subsection{Applying an ecological perspective}

When working with refugees, Kira and Tummala state the importance for social workers to adopt an ecological model of recovery [27]. This provides a holistic approach in addressing the differing needs refugees face after resettlement. Informed by Urie Brofenbreener's ecological perspective (Bronfenbrennerm 1979) it suggests that human development is shaped by several systems or contexts, and social workers can then understand how an individual is impacted by their family, community, and environment [28]. This will better support refugees themselves when identifying the quality of interactions, they have with their micro, meso and macro systems.

In a German study with Syrian refugee women in identifying and understanding the barriers in accessing mental health care, McLeroy's adaptation of the socio-ecological model (SEM) was used as a framework to situate the barriers on the various SEM layers spanning from individual to policy level, explaining specifically how different individual and environmental factors determine health behavior in individuals [29]. The SEM is a useful framework because it conceptualizes human development by placing the individual into the centers of circles surrounding it, highlighting the interrelationship of multiple determinants of development and interactions at the personal, relational and collective levels within this dynamic socio-ecological environment [30,31]. With this framework, there is an assumption that individual decisions and behaviors are determined by reciprocal interactions within and between the social and physical environment of individuals. Simultaneously, individuals contribute to their social ecology in terms of constructing norms, beliefs, and culture across multiple macro-systems [30]. Intrinsically, the SEM states that individual level behavior is shaped by multiple environmental factors and vice-versa, recognizing the important social environmental and biological factors that either cultivate or inhibit individual attitudes and behaviors [32].

Addressing the ecological perspective for mental health intervention is a critical component for cultural appropriate and sustainable practice, where the micro-system considers the individual and family relationship and interactions. The meso-system acknowledges the individual and what supports they have or lack within the community. The macro-system focuses on the programs, assessments and policies that affect the lives of refugees and their families.

\section{Materials and Methods}

\subsection{Design}

The systematic review followed the guidelines of the Preferred Reporting Items for Systematic Reviews and Meta-Analysis (PRISMA) statement [33]. The PRISMA statement includes 27-item checklist which assures the transparency, iteration, and complete reporting for systematic reviews.

\subsection{Search Strategy}

The search was conducted in ScienceDirect, Scopus, PubMed, Medline, Web of Science, ProQuest, Wiley, and Elsevier in December 2020 to January 2021. The electronic databases were searched using the below terms identified form the title, abstract, keywords, or headings: ('mental health' OR 'refugee' OR 'asylum seeker' OR 'risk factors' OR 'protective factors' AND 'predictors' OR 'measurements' OR 'determinants'. The search terms 
were adapted from the previous review studies with a similar purpose. We also manually searched reference lists of relevant articles to identify additional publications. Finally, references of all included studies were listed to eliminate the duplications and resolve proper reporting guidelines for the selected articles. different synonyms were used for the search.

\subsection{Eligibility Criteria}

All quantitative and qualitative studies were considered for the systematic review. Studies were included if they (1) Evaluated overall mental health issues of the forced migrants; (2) assessed any association between depression, post-traumatic stress disorder, mental disorder determinants and migratory backgrounds; (3) tested any theoretical framework related to mental health (4) compared mental health issues or its determinants between particular migrant populations or with general populations in the host societies; (5) conducted a literature review, systematic review or meta-analysis/ synthesis on mental health determinants of forced migrants e.g. refugees, and asylum seekers. Studies published in full in peer-reviewed journals between January 2015 and January 2021 and in English language were included. Studies with less than 50 participants in quantitative articles were excluded. We eliminated dissertations, conference abstracts and organizational reports from the review. Different stages of sampling are provided (Figure 1). We reviewed prevalence rates, risk, and protective factors for the 3 common mental health issues among refugees and asylum-seekers including post-traumatic stress disorder, depression, and mental disorder. These three negative mental health outcomes are reportedly frequent among refugees and asylum seekers from the initial pre-migration stages to host country resettlement and post-resettlement [34]. 
Identification of studies via databases and registers

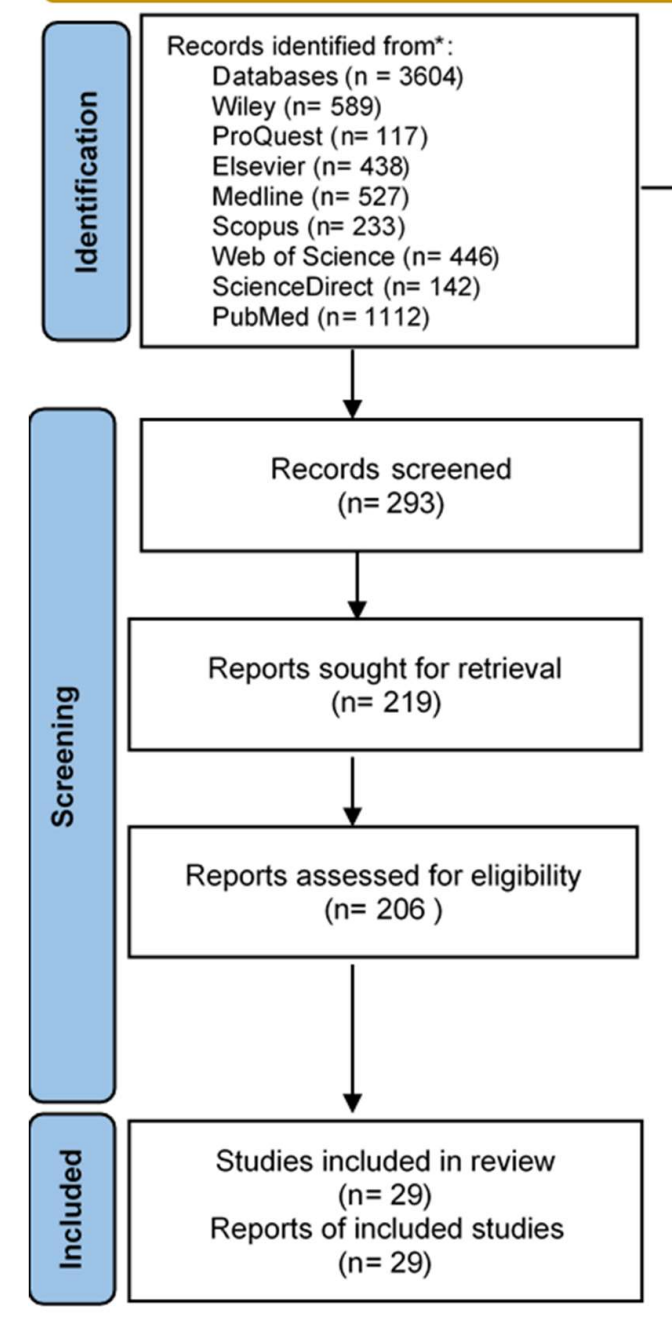

Records removed before screening:

Duplicate records removed $(n=$ 364)

Records marked as ineligible by automation tools $(n=2902)$

Records removed for other reasons $(n=45)$

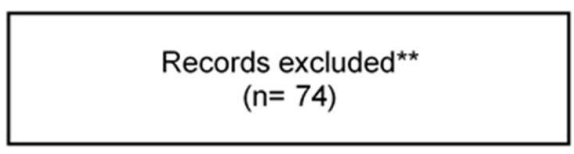

Figure 1Flow diagram depicting different stages of sampling [35]

\subsection{Selection of the Studies}

Titles and abstracts of the studies derived from the databases were reviewed twice by two independent authors before proceeding to the next stage of the review. To solve the disagreements between authors in eliminating or including papers in the review process, third author was consulted. Full texts of all included studies were checked twice according to the eligibility criteria by the authors and disagreements were resolved.

\subsection{Data Extraction}

To ease the review process, data were entered into a previously prepared data extraction sheet. In the data extraction sheet we itemized (1) study characteristics including author names, publish year, and country; (2) sampling method and target population; (3) methods of assessment and analysis, and (4) key findings including risk and protective factors for mental health. Characteristics of the included qualitative and quantitative studies are consecutively shown in Table 1 and Table 2. 
Table 1. Characteristics of the included qualitative studies

\begin{tabular}{|c|c|c|c|c|c|c|c|c|}
\hline $\begin{array}{l}\text { Study } \\
\text { ID }\end{array}$ & $\begin{array}{l}\text { Author (s) } \\
\text { Country }\end{array}$ & $\begin{array}{l}\text { Time } \\
\text { period }\end{array}$ & $\begin{array}{l}\text { Sampling } \\
\text { method }\end{array}$ & $\begin{array}{c}\text { Interview } \\
\text { method }\end{array}$ & $\begin{array}{c}\text { Study } \\
\text { population }\end{array}$ & $\begin{array}{c}\text { Sample } \\
\text { size }\end{array}$ & $\begin{array}{l}\text { Age } \\
\text { range }\end{array}$ & $\begin{array}{l}\text { Protective } \\
\text { factors }\end{array}$ \\
\hline 1 & $\begin{array}{l}\text { Affleck et } \\
\text { al, } 2018 \\
{[36]} \\
\text { Canada }\end{array}$ & $\begin{array}{l}2012- \\
2016\end{array}$ & $\begin{array}{l}\text { Convenience } \\
\text { Snowball }\end{array}$ & $\begin{array}{l}\text { Semi- } \\
\text { structured }\end{array}$ & $\begin{array}{l}\text { Sri Lankan } \\
\text { Tamil } \\
\text { refugee } \\
\text { men }\end{array}$ & 33 & $20-60$ & $\begin{array}{l}\text { Participating in } \\
\text { religious rituals, } \\
\text { meditation, } \\
\text { adherence to } \\
\text { familial and } \\
\text { community duties }\end{array}$ \\
\hline
\end{tabular}

\begin{tabular}{|c|c|c|c|c|c|c|c|c|}
\hline 2 & $\begin{array}{l}\text { Alemi et al, } \\
2016 \text { [37] } \\
\text { USA }\end{array}$ & $\begin{array}{l}\text { July- } \\
\text { October } \\
2012\end{array}$ & Snowball & $\begin{array}{l}\text { Semi- } \\
\text { structure }\end{array}$ & $\begin{array}{l}\text { Afghan } \\
\text { refugees \& } \\
\text { asylum- } \\
\text { seekers }\end{array}$ & $\begin{array}{l}18= \\
(11) \\
\text { males, } \\
(7) \\
\text { females }\end{array}$ & $36-71$ & $\begin{array}{l}\text { Family } \\
\text { reunification, } \\
\text { community support, } \\
\text { prayers, } \\
\text { successfulness of } \\
\text { the children }\end{array}$ \\
\hline
\end{tabular}

\begin{tabular}{|c|c|c|c|c|c|c|c|c|}
\hline 3 & $\begin{array}{l}\text { Im, } \\
\text { Ferguson, } \\
\text { and Hunter } \\
2017 \text { [38] } \\
\text { Kenya }\end{array}$ & Unknown & Purposive & $\begin{array}{l}\text { Semi- } \\
\text { structure } \\
\text { and focus } \\
\text { groups }\end{array}$ & $\begin{array}{l}\text { Somali } \\
\text { refugees }\end{array}$ & $\begin{array}{l}15 \text { key } \\
\text { informa } \\
\text { nts, } 31 \\
\text { focus } \\
\text { group } \\
\text { particip } \\
\text { ants }\end{array}$ & $18-56$ & $\begin{array}{l}\text { Counseling, job } \\
\text { opportunities, } \\
\text { mindfulness } \\
\text { exercises, } \\
\text { socializing, prayers, } \\
\text { personal reflections, }\end{array}$ \\
\hline 4 & $\begin{array}{l}\text { Omar, } \\
\text { Kuay, and } \\
\text { Tuncer } \\
2017 \text { [39] } \\
\text { Astralia }\end{array}$ & $\begin{array}{l}2013- \\
2014\end{array}$ & Purposive & $\begin{array}{l}\text { Semi- } \\
\text { structure } \\
\text { and focus } \\
\text { groups }\end{array}$ & $\begin{array}{l}\text { Muslim } \\
\text { refugees } \\
\text { from } \\
\text { Somalia, } \\
\text { Eritrea, } \\
\text { Ethiopia, } \\
\text { and } \\
\text { Djibouti }\end{array}$ & 36 & $18-60$ & $\begin{array}{l}\text { Prayer, confidence } \\
\text { in Allah, family and } \\
\text { community support, } \\
\text { cultural } \\
\text { rehabilitation } \\
\text { methods (visiting } \\
\text { African } \\
\text { environment) }\end{array}$ \\
\hline
\end{tabular}

\begin{tabular}{|c|c|c|c|c|c|c|c|c|}
\hline 5 & $\begin{array}{l}\text { Savic et al. } \\
2016[40] \\
\text { Australia }\end{array}$ & Unknown & $\begin{array}{l}\text { Semi- } \\
\text { structure }\end{array}$ & $\begin{array}{l}\text { Refugees, } \\
\text { health } \\
\text { service } \\
\text { providers }\end{array}$ & 45 & +18 & $\begin{array}{l}\text { Community } \\
\text { counselling, } \\
\text { community supports }\end{array}$ & $\begin{array}{l}\text { Pre-migration experiences e.g., } \\
\text { exposure to violence, rape, loss } \\
\text { of loved ones, distrust of } \\
\text { western mental health services, } \\
\text { medicalized nature of western } \\
\text { mental health services, } \\
\text { stigmatization of mental health } \\
\text { issues, incompatibility of needs } \\
\text { and offered mental health } \\
\text { services }\end{array}$ \\
\hline 6 & $\begin{array}{l}\text { Poudel- } \\
\text { Tandukar et } \\
\text { al., } 2019 \\
\text { [41] } \\
\text { USA }\end{array}$ & $\begin{array}{l}\text { Convenience } \\
\text { Snowball }\end{array}$ & $\begin{array}{l}\text { Focus } \\
\text { groups }\end{array}$ & $\begin{array}{l}\text { Bhutanese } \\
\text { refugees }\end{array}$ & 67 & +18 & $\begin{array}{l}\text { Community } \\
\text { supports, sharing } \\
\text { problems with } \\
\text { peers, cultural } \\
\text { empowerments and } \\
\text { raising awareness }\end{array}$ & $\begin{array}{l}\text { language learning issues, } \\
\text { decreased support systems, } \\
\text { increased responsibilities in the } \\
\text { host countries, socio-economic } \\
\text { disadvantages, lack of access to } \\
\text { services, change in family }\end{array}$ \\
\hline
\end{tabular}

Inability to protect family members, inability to fulfill cultural duties, inability to perform social roles, Un/Uderemployment, inner-family conflicts

Imprisonment of family members, arbitrary home invasions, constant fears of being killed or maltreated, harsh fleeing path, abrupt separation from family-cultural adjustment issues, fear of deportation, language learning barriers, intergenerational challenges with children, unemployment, cultural insensitiveness of social services, losing culture and identity lack of educational opportunities, work and decent life, isolation, daily physical health issues, distressing events, unfulfilled desires. insufficient means of support, losing loved ones, community violence

Unemployment, underemployment, intercultural conflicts in the host country, religious boundaries, inability to support families overseas, lack of mental health literacy, doubt in identifies treatment strategies (faith-based) treatments,
Pre-migration experiences e.g., exposure to violence, rape, loss , distrust of medicalized nature of western mental health services, stigmatization of mental health ef need services

language learning issues, decreased support systems, ased responsibilities in the disadvantages, lack of access to , change in family 
dynamics, discrimination,

stigmatization,

\begin{tabular}{|c|c|c|c|c|c|c|c|c|}
\hline 7 & $\begin{array}{l}\text { Yaser et al., } \\
2016[42] \\
\text { Australia }\end{array}$ & $\begin{array}{l}2015- \\
2016\end{array}$ & $\begin{array}{l}\text { Convenience } \\
\text { Snowball }\end{array}$ & $\begin{array}{l}\text { Semi- } \\
\text { structure }\end{array}$ & $\begin{array}{l}\text { Afghan } \\
\text { Refugees }\end{array}$ & $\begin{array}{l}150= \\
(74) \\
\text { males, } \\
(76) \\
\text { females }\end{array}$ & $20-45$ & $\begin{array}{l}\text { Improving diet or exercise, raising self-awareness, } \\
\text { psychotherapy focusing on the past events, finding new } \\
\text { hobbies, physical activity, socializing, meditation, herba } \\
\text { medication, }\end{array}$ \\
\hline 8 & $\begin{array}{l}\text { Yassin et } \\
\text { al., } 2017 \\
\text { [43] } \\
\text { Lebanon }\end{array}$ & $\begin{array}{l}\text { August- } \\
\text { November } \\
2015\end{array}$ & Convenience & $\begin{array}{l}\text { Semi- } \\
\text { structure }\end{array}$ & $\begin{array}{l}\text { Palestinian } \\
\text { refugees }\end{array}$ & $\begin{array}{l}49= \\
(28) \\
\text { refugee } \\
\text { s, (11) } \\
\text { service } \\
\text { provide } \\
\text { rs, } \\
(10) \\
\text { local } \\
\text { commu } \\
\text { nity } \\
\text { represe }\end{array}$ & $24-58$ & $\begin{array}{l}\text { Easy access to services, home visits of social workers, } \\
\text { individual based treatments, raising awareness of } \\
\text { refugees about mental health, access to the history of } \\
\text { mental health issues of refugees, sustainability of the } \\
\text { mental health services, prompt access to mental health } \\
\text { services } \\
\text { upon entry to the country }\end{array}$ \\
\hline
\end{tabular}

\begin{tabular}{|c|c|c|c|c|c|c|c|c|}
\hline 9 & $\begin{array}{l}\text { Yu et al., } \\
2018 \text { [44] } \\
\text { South } \\
\text { Korea }\end{array}$ & $\begin{array}{l}\text { July- } \\
\text { August } \\
2013\end{array}$ & Purposive & $\begin{array}{l}\text { Semi- } \\
\text { structure }\end{array}$ & $\begin{array}{l}\text { North } \\
\text { Korean } \\
\text { refugees }\end{array}$ & $\begin{array}{l}10= \\
(8) \\
\text { females } \\
(2) \\
\text { males }\end{array}$ & $20-69$ & $\begin{array}{l}\text { Prompt treatment } \\
\text { upon arrival, raised } \\
\text { self-awareness, } \\
\text { counselling }\end{array}$ \\
\hline
\end{tabular}

Absence of mental health awareness, issues of survival, stigmatization, lack of access to mental health services, trauma experienced during the escape, cultural encounter shocks, isolation and lose identity Stress of deportation, asylum seeking process, sense of powerlessness, confinement in the detention centers, inadequate supports, feelings of retraumatization, un/underemployment, high Refugees Vitale, and Ryde, 2016 [45] Purposive Semistructure

Sudan-

Iran-

Eritrea-

Morocco-

9 $29-62$

Voluntary works, expectations upon arrival, U.K.

Somalia) target oriented work trainings community, inability to establish inadequate means of living, lack of practical information, lack of integration in the host new networks, cultural barriers, inadequate mental health services, no opportunities to be an active citizen

Table 2. Characteristics of the included quantitative studies

\begin{tabular}{|c|c|c|c|c|c|c|c|}
\hline $\begin{array}{c}\text { Study } \\
\text { ID }\end{array}$ & $\begin{array}{l}\text { Author (s) } \\
\text { Country }\end{array}$ & $\begin{array}{l}\text { Time } \\
\text { period }\end{array}$ & Methodology & $\begin{array}{c}\text { Study } \\
\text { population }\end{array}$ & $\begin{array}{c}\text { Sample } \\
\text { size }\end{array}$ & $\begin{array}{l}\text { Protective } \\
\text { factors }\end{array}$ & $\begin{array}{l}\text { Risk } f \\
\text { factors }\end{array}$ \\
\hline 11 & $\begin{array}{l}\text { Poole et al, } 2018[46] \\
\text { Greece }\end{array}$ & $\begin{array}{l}\text { January } \\
2017\end{array}$ & Survey & $\begin{array}{l}\text { Syrian Refu- } \\
\text { gees }\end{array}$ & 135 & Not mentioned & $\begin{array}{l}\text { Gender (being women), } \\
\text { having children, increased } \\
\text { time in the asylum process }\end{array}$ \\
\hline 12 & $\begin{array}{l}\text { Chung et al, } 2018 \text { [47] } \\
\text { Turkey, Sweden }\end{array}$ & 2017 & Survey & $\begin{array}{l}\text { Syrian Refu- } \\
\text { gees }\end{array}$ & $\begin{array}{l}1197(482 \\
\text { females, }\end{array}$ & $\begin{array}{l}\text { Age (younger refugees } \\
\text { are more resilient), stable }\end{array}$ & $\begin{array}{l}\text { Location of resettlement, } \\
\text { came alone of with family, }\end{array}$ \\
\hline
\end{tabular}


715 relationship with com- having less community net-

males) munity, employment, so- works

cial networks

\begin{tabular}{|c|c|c|c|c|c|c|c|}
\hline 13 & $\begin{array}{l}\text { Hocking, \& Sundram } 2015 \\
{[48]} \\
\text { Australia }\end{array}$ & $\begin{array}{l}\text { Not men- } \\
\text { tioned }\end{array}$ & Survey & $\begin{array}{l}\text { Refugees ( } \mathrm{n}= \\
33 \text { ) and asy- } \\
\text { lum seekers } \\
(\mathrm{n}=98) \text { form } \\
\text { Zimbabwe, } \\
\text { Afghanistan, } \\
\text { Iran, Iraq, } \\
\text { Lebanon, Pa- } \\
\text { kistan, Sri } \\
\text { Lanka }\end{array}$ & 131 & $\begin{array}{l}\text { Social networks, em- } \\
\text { ployment }\end{array}$ & $\begin{array}{l}\text { Gender, age, social isola- } \\
\text { tion, low socio-economic } \\
\text { status, family separation, } \\
\text { unclear residence status, } \\
\text { detention experience, } \\
\text { mode of arrival }\end{array}$ \\
\hline 14 & $\begin{array}{l}\text { Campbel et al, } 2018 \text { [49] } \\
\text { U.K. }\end{array}$ & $2005-2007$ & $\begin{array}{l}\text { Longitudinal } \\
\text { survey }\end{array}$ & Refugees & 5678 & $\begin{array}{l}\text { Involvement in commu- } \\
\text { nity networks, easy ac- } \\
\text { cess to healthcare ser- } \\
\text { vices }\end{array}$ & $\begin{array}{l}\text { unemployment, language } \\
\text { barriers, unsatisfactory } \\
\text { accommodation, being } \\
\text { victims of discrimination, } \\
\text { infrequent contact with } \\
\text { relatives }\end{array}$ \\
\hline 15 & $\begin{array}{l}\text { Şimşek et al, } 2018 \text { [50] } \\
\text { Turkey }\end{array}$ & 2015 & $\begin{array}{l}\text { Cross- } \\
\text { sectional, } \\
\text { interview }\end{array}$ & Refugees & $\begin{array}{l}458= \\
\text { females, } \\
15-49 \\
\text { years old }\end{array}$ & $\begin{array}{l}\text { social support, commu- } \\
\text { nity-based and culturally } \\
\text { sensitive health educa- } \\
\text { tion programs, inclusion } \\
\text { of mental health care } \\
\text { within basic primary care } \\
\text { services }\end{array}$ & $\begin{array}{l}\text { Household size, difficulty } \\
\text { to get health services }\end{array}$ \\
\hline 16 & $\begin{array}{l}\text { Dietrich et al, } 2019 \text { [51] } \\
\text { Germany }\end{array}$ & & $\begin{array}{l}\text { Interview, sur- } \\
\text { vey }\end{array}$ & $\begin{array}{l}\text { Refugees } \\
\text { from Syria } \\
\text { and Iraq }\end{array}$ & 2057 & $\begin{array}{l}\text { social support and net- } \\
\text { works, higher education }\end{array}$ & $\begin{array}{l}\text { Experience of violence, } \\
\text { poor housing and sense of } \\
\text { insecurity }\end{array}$ \\
\hline 17 & $\begin{array}{l}\text { Georgiadou et al, } 2018 \\
{[52]} \\
\text { Germany }\end{array}$ & $\begin{array}{l}\text { Not } \\
\text { mentioned }\end{array}$ & Survey & $\begin{array}{l}\text { Syrian } \\
\text { refugees }\end{array}$ & 518 & Inclusive welfare system & $\begin{array}{l}\text { Death of a loved one, age, } \\
\text { shorter validity of resi- } \\
\text { dence permit, longer dura- } \\
\text { tion of asylum procedure, } \\
\text { poor economic conditions }\end{array}$ \\
\hline 18 & $\begin{array}{l}\text { Schweitzer et al, } 2018 \text { [53] } \\
\text { Australia }\end{array}$ & 2013- 2015 & $\begin{array}{l}\text { Cross-sectional } \\
\text { survey }\end{array}$ & Refugees & 104 & $\begin{array}{l}\text { Access to health and } \\
\text { welfare services, im- } \\
\text { proved pre-arrival infor- } \\
\text { mation about the host so- } \\
\text { cieties }\end{array}$ & $\begin{array}{l}\text { Traumatic experiences, } \\
\text { racial discrimination }\end{array}$ \\
\hline 19 & $\begin{array}{l}\text { Segal et al, } 2018 \text { [54] } \\
\text { Lebanon }\end{array}$ & 2012- 2013 & $\begin{array}{l}\text { Survey, } \\
\text { interview }\end{array}$ & $\begin{array}{l}\text { Palestinian } \\
\text { refugees }\end{array}$ & 254 & $\begin{array}{l}\text { Housing stability, eco- } \\
\text { nomically gainful em- } \\
\text { ployment, social net- } \\
\text { works, immediate access } \\
\text { to mental health clinics }\end{array}$ & $\begin{array}{l}\text { Traumatic experiences, } \\
\text { human right violations }\end{array}$ \\
\hline
\end{tabular}




\begin{tabular}{|c|c|c|c|c|c|c|c|}
\hline $20^{*}$ & $\begin{array}{l}\text { Grupp et al, } 2018 \text { [55] } \\
\text { Germany }\end{array}$ & $\begin{array}{l}\text { Not } \\
\text { mentioned }\end{array}$ & $\begin{array}{l}\text { Survey, focus } \\
\text { groups }\end{array}$ & $\begin{array}{l}\text { African } \\
\text { refugees, } \\
\text { German } \\
\text { population }\end{array}$ & 239 & $\begin{array}{l}\text { Praying, ability to fulfill } \\
\text { religious rituals }\end{array}$ & $\begin{array}{l}\text { Isolation, Intergenera- } \\
\text { tional conflicts }\end{array}$ \\
\hline 21 & $\begin{array}{l}\text { Kandemir et al, } 2018 \text { [56] } \\
\text { Turkey }\end{array}$ & $\begin{array}{l}\text { Not } \\
\text { mentioned }\end{array}$ & Survey & $\begin{array}{l}\text { Syrian } \\
\text { refugees }\end{array}$ & 355 & $\begin{array}{l}\text { Social and welfare sup- } \\
\text { ports, education, Social } \\
\text { networks }\end{array}$ & $\begin{array}{l}\text { Traumatic experiences, } \\
\text { gender, proper housing, } \\
\text { enough food, experiences } \\
\text { of discrimination and rac- } \\
\text { ism }\end{array}$ \\
\hline 22 & $\begin{array}{l}\text { Shawyer et al, } 2017 \text { [57] } \\
\text { Australia }\end{array}$ & $\begin{array}{l}\text { Not } \\
\text { mentioned }\end{array}$ & Survey & $\begin{array}{l}\text { Refugees, and } \\
\text { asylum- } \\
\text { seekers }\end{array}$ & 135 & $\begin{array}{l}\text { Culturally responsive } \\
\text { mental health services, } \\
\text { early mental health as- } \\
\text { sessments and treatments }\end{array}$ & $\begin{array}{l}\text { Human right violations, } \\
\text { stressful migration experi- } \\
\text { ences, cultural and lan- } \\
\text { guage barriers to get ac- } \\
\text { cess to mental health ser- } \\
\text { vices, country of origin }\end{array}$ \\
\hline 23 & $\begin{array}{l}\text { Slewa-Younan et al, } 2017 \\
\text { [58] } \\
\text { Australia }\end{array}$ & 2013 & $\begin{array}{l}\text { Interview, } \\
\text { survey }\end{array}$ & $\begin{array}{l}\text { Afghan } \\
\text { refugees }\end{array}$ & 150 & $\begin{array}{l}\text { Early intervention pro- } \\
\text { grams }\end{array}$ & $\begin{array}{l}\text { Past traumatic experi- } \\
\text { ences }\end{array}$ \\
\hline 24 & $\begin{array}{l}\text { Tinghög et al, } 2017 \text { [59] } \\
\text { Sweden }\end{array}$ & 2011-2013 & $\begin{array}{l}\text { Cross-sectional } \\
\text { survey }\end{array}$ & $\begin{array}{l}\text { Syrian } \\
\text { refugees }\end{array}$ & 1215 & $\begin{array}{l}\text { Reunion with family } \\
\text { members }\end{array}$ & $\begin{array}{l}\text { Gender, traumatic experi- } \\
\text { ences, lower education, } \\
\text { Isolation in host society, } \\
\text { ethnic discrimination }\end{array}$ \\
\hline 25 & $\begin{array}{l}\text { Lillee et al, } 2015 \text { [60] } \\
\text { Australia }\end{array}$ & $\begin{array}{l}\text { Not } \\
\text { mentioned }\end{array}$ & Survey & Refugees & 300 & $\begin{array}{l}\text { Routine use of mental } \\
\text { health services, cultur- } \\
\text { ally sensitive mental } \\
\text { health care }\end{array}$ & $\begin{array}{l}\text { Marital status, having } \\
\text { more children, past trau- } \\
\text { matic events, stigmatiza- } \\
\text { tion, }\end{array}$ \\
\hline 26 & $\begin{array}{l}\text { Leiler et al, } 2019 \text { [61] } \\
\text { Sweden }\end{array}$ & $\begin{array}{l}\text { Not } \\
\text { mentioned }\end{array}$ & $\begin{array}{l}\text { Survey, } \\
\text { interview }\end{array}$ & $\begin{array}{l}\text { refugees and } \\
\text { asylum } \\
\text { seekers }\end{array}$ & 510 & $\begin{array}{l}\text { Safety, access to heath } \\
\text { care upon arrival, short- } \\
\text { ening asylum process }\end{array}$ & $\begin{array}{l}\text { unclear residence permit } \\
\text { status, poor neighbor- } \\
\text { hoods }\end{array}$ \\
\hline 27 & $\begin{array}{l}\text { Rizkalla and Segal, } 2018 \\
{[62]} \\
\text { Jordan }\end{array}$ & 2014- 2016 & Survey & $\begin{array}{l}\text { Syrian } \\
\text { refugees }\end{array}$ & 250 & $\begin{array}{l}\text { Active NGOs, refugee- } \\
\text { friendly mental health } \\
\text { services }\end{array}$ & $\begin{array}{l}\text { Traumatic events, un/ un- } \\
\text { deremployment, poor } \\
\text { neighborhood disad- } \\
\text { vantages }\end{array}$ \\
\hline 28 & $\begin{array}{l}\text { Acarturk et al, } 2018 \text { [63] } \\
\text { Turkey }\end{array}$ & $\begin{array}{l}\text { March- } \\
\text { May } 2013\end{array}$ & $\begin{array}{l}\text { Survey, } \\
\text { interview }\end{array}$ & $\begin{array}{l}\text { Syrian } \\
\text { refugees }\end{array}$ & 781 & $\begin{array}{l}\text { Inclusive public health } \\
\text { policies }\end{array}$ & $\begin{array}{l}\text { Traumatic events, losing } \\
\text { sources of income, gen- } \\
\text { der, living in camps }\end{array}$ \\
\hline 29 & $\begin{array}{l}\text { Pandya, } 2018[64] \\
\text { Europe }\end{array}$ & $\begin{array}{l}\text { Not } \\
\text { mentioned }\end{array}$ & Survey & Refugees & 4504 & $\begin{array}{l}\text { Voluntary participation } \\
\text { in mental health pro- } \\
\text { grams, self-practice will- } \\
\text { ingness, spirituality, } \\
\text { group-based interven- } \\
\text { tions }\end{array}$ & $\begin{array}{l}\text { Country of origin, refugee } \\
\text { status duration, gender, } \\
\text { past traumatic experi- } \\
\text { ences }\end{array}$ \\
\hline
\end{tabular}


The quality of the eligible studies e.g. heterogeneity and variability in the design, was assessed by the GRADE approach for grading the quality of evidence and the strength of the recommendations in systematic reviews. GRADE approach was developed to improve the transparency of the process of systematic reviews and presenting a logical support or evidence and recommendations [65-67]. According to GRADE guidance, five main quality factors of evidence are as follows: (1) risk of biases, (2) inconsistency of results, (3) indirectness of evidence, (4) impression, and (5) publication bias. Most of the selected studies were non-experimental and their quality were ranked as low. Articles were excluded in this review if they were seriously lacking experimental feature. The main author doubly checked selected studies to assess the quality. A second author checked for the precision of the assessment. Disagreements were resolved through discussion.

\subsection{Data Analysis}

Due to the heterogeneity of the measurements, methods, and theoretical bases of the selected studies, utilizing statistical methods to combine data for further analysis were impossible. Moreover, study countries, population characteristics and data collection methods were different. As a result, data were narratively synthesized in the systematic review. Selected studies were categorized by the origin of the sample population, mental health risk factors and protective factors, and the prevalence rates of the identified mental health issues e.g. depression, post-traumatic stress disorder, and mental disorder. Within each category, consistency, or contradictions regarding the results of the selected studies were synthesized. Reasons for conflicting results in the prevalence rates, or risks and protective factors for mental health were interpreted according to the evidence and study characteristics e.g. methodological errors, sampling bias, or measure shortcomings.

\section{Findings}

\subsection{Prevalence Rates}

Prevalence rates of post-traumatic stress disorder among refugees and asylum seekers in the included quantitative studies were substantially heterogeneous with a range fluctuating between 5.1\% [54] to 83.4\% [63]. A critical factor in understanding conditions impacting post-traumatic stress disorder in refugee population is the country of origin and the migration pathway. As Pandya contended in a study of five refugee groups from Syria, Iraq, Afghanistan, Eritrea, and Somalia, the country of origin was a significant determinant in PTSD, depression, and mental disorder prevalence rates [53,64]. Refugees studied by Segal were Palestinians who had lived in Lebanese camps for 21 years [54]. They appeared to have less problems in terms of learning language, developing social networks, and integrating into the host society simply because of the very similar culture and the official Arabic language in the host country in Lebanon. They had fewer PTSD symptoms than participants in similar studies, but their general mental health was critical. However, Leiler et.al reported that levels of PTSD symptoms among refugees were about twice as high as in global general population studies [61]. Lillee et.al found significantly higher PTSD prevalence rates among refugees than among the general population [60].

Depression prevalence rates varied less than post-traumatic stress disorder, however, it was still impossible to attain an understanding of the situation. Major depressive disorder ranged from $14.5 \%$ [52] to 53.1\% [48], which is substantially higher than the rates among the general population of the host society. Leiler et.al concluded that the prevalence of depressive symptoms was five times higher in the sample population than in the Swedish general population [61]. Georgiadou et al, investigated PTSD symptoms among settled refugees with residence permits in Germany [52], while the sample population in Hocking and Sundram's study investigated asylum-seekers in Melbourne, Australia, without residence permits [48]. This reason may justify the gap between the findings of the two studies and highlights the importance of having a residence permit [52].

Mental health prevalence rates varied from 35.7\% [60] to 55\% [59] in the selected refugee population. The country of origin was again found to be a significant mental 
health determinant and could be a reason for the differences in the reports of prevalence rates of general mental health [64].

\subsection{Comparing Risk and Protective Factors across Mental Health Diagnosis}

The evidence presented in this systematic review supports the findings of previous studies that mental health issues of vulnerable populations are multidimensional, and widely measured outcome of health issues, yet of great concerns. However, the measures and perspectives that attempted to explain mental health issues are top-down and academic-marketing oriented, and less of them were supported to really explain refugee's points of views.

Socio-demographic factors in this literature review appeared to reflect the findings of previous research. Women were at greater risks of developing poorer mental health than men, and poor mental health was associated with having more children $[46,54]$. Due to the possibility of adapting to a new environment and behavioral resilience, young refugees could find adjustment to a new culture easier compared to the older age refugees [52]. The country of origin and the reasons for migration appeared to be positively associated with mental health, post-traumatic stress disorder, and depression symptoms [64]. Low socioeconomic and educational levels were strongly associated with mental health outcomes, as poorly educated refugees with lower incomes were at greater risks of adverse mental health outcomes [38,50,51]. However, it is proven that recently resettled refugees with higher education and socioeconomic backgrounds in their home countries were at greater risks of developing mental health issues during the period leading up to the outcome of their asylum application [68]

Unemployment was another important risk factor that was referred to across the selected studies $[48,49,51,54]$. However, the causation of the link between unemployment (or under-employment) and mental health remains contentious [51,69]. Campbell et al. referred to the direct effect of unemployment on mental health issues in which unemployed people suffer negative side effects in daily life [49]. Unemployment may result in financial problems and loss of self-esteem, loss of social networks and social participation, and may increase the risk of dangerous behaviors, such as smoking, drinking, and drug abuse [39].

Another common risk factor was the effect of housing quality. Some refugees were accommodated outside of urban areas in poor quality collective shelters. Dissatisfaction with accommodation resulted in isolation and low self-esteem and both factors endanger the mental health of refugees and asylum seekers $[49,70]$. Accommodation policies of the host countries have been a growing area of controversy. Phillimore and Goodson long before the 2015 refugee crisis contended that policies for accommodating refugees in dispersal areas may result in higher levels of unemployment. They concluded that these processes together exacerbate the general levels of social exclusion in the host societies [70].

Infrequent contact and interaction with relatives and friends found to be associated with poorer mental health outcomes [47]. Development of new social networks in host countries found to be an offsetting element [50]. The importance of language acquisition for social interaction was highlighted. Involvement in social activities and community networks requires acceptable knowledge of language skills [49]. Without linguistic skills, no connection, and consequently, no integration is possible. This problem was mostly common among older refugees. The potential for learning a new language decreases as age raises [69]. Thus, social isolation, because of not having enough language skills, was strongly associated with negative mental health issues.

Perceived discrimination and prejudice appeared to be associated with poorer mental health. Victims of discrimination and physical violence report that they suffer from adverse well-being feelings [71]. They constantly worry about the recurrence of such incidents and feel angry. This makes these victims isolated as time passes on, and in extreme cases, an individual may respond with violent behaviors [72].

Selected qualitative studies in the current review provided more in-depth view of the risks and protective factors for the mental health issues. In terms of protective factors, 
community supports, and social networks of refugees seemed to play a crucial role in dealing with mental health issues [37-39,41,72]. According to the findings of Affleck et al., community representatives of Sri Lankan Tamil refugees, were actively screening mental health of each members of the community and in doing so, those who were recognized suffering from various mental health issues, were closely taken care and treated according to the traditional practices [36]. This can be a good example for a sustainable mental health program that shows how to use community potentials to manage its member's well-being, and health.

Cultural empowerments of service providers and raising awareness of the people in the host countries through sustainable intervention plans were another effective protective factor for the mental health of refugees [41]. Cultural awareness specially in the side of social workers and service providers creates mutual understanding of refugee's various needs. The compatibility of the services with the cultural beliefs and expectations of the stakeholders, as Pandya mentions, raises voluntary participation and self-practice willingness towards mental health services among refugees and asylum seekers [64].

Considering all risks and protective factors for the mental health of forced migrants, qualitative studies proved to be more detailed and reliable. Especially when it comes to the practice research and seeking sustainable solutions for the issues of forced migrants. Selected qualitative studies in this review also merited acquiring bottom-up approaches in which, refugees and asylum seekers are actively participated in the research process. This is in line with our contends on the benefits of the ecological perspective when dealing with vulnerable populations.

\section{Discussion}

\subsection{Promoting mental health care - implications for sustainable social work practice}

The bold fact of leaving one's home forever and adapting to a new environment and culture is highly stressful and when associated with lack of social integration and unemployment, the lived experience of stress increases in a stepwise manner [73]. The high prevalence of mental disorders associated with refugees can pose significant challenges such as the impact of pre-migration traumas on mental health in the settlement context. Very few studies have found that mental health interventions such as narrative exposure therapy have been successful. Sometimes therapies can focus on diagnostic features of PTSD whilst overlooking aspects such as relationships and a sense of meaning [74-76]. Interventions should include social integration, facilitate access to care as well as fostering engagement to promote and provide good mental healthcare to these groups in a culturally appropriate, holistic, and sensitive manner.

There are certain gaps in the research identifying what factors in specific contexts would help. Nevertheless, the literature has reported that certain interventions are more ecologically valid. Naturalistic mental health interventions could be seen as alternatives for refugees where treatment is provided in the context of an existing service including social and medical services [75]. Interventions that utilize available information and communication technologies (ICTs) would be seen to be advantageous. One of the most salient mental health issues results from refugees missing their loved ones and being concerned about their well-being and ICTs has not only facilitated refugees' well-being by facilitating their journey to their destination but also it helps them find out critical information postarrival of their host country. Most importantly it bridges the gap between separated families. Therefore, it would be practical and innovative to use communication technologies that refugees already widely use that would even further facilitate diagnostic testing and address language barriers.

The issue of mental health in the Refugee context is therefore a complex one and because of the synergistic relationship between refugee mental health and the broader social context, it is important that more research is conducted identifying who benefits from certain interventions and at what point in their integration trajectory would be most effective. This knowledge is relevant for social work and policymakers to design and 
deliver sustainable mental health care services with an ecological perspective harnessing the practical tools that refugees already possess. This can ultimately enhance the social wellbeing of this frequently marginalized population and it is also relevant to the whole process of successful integration which ultimately benefits economies of the receiving countries.

\subsection{Limitations}

Systematic reviews are useful methods for informing policy and practice and was chosen in this study because it allows us to combine various conceptualized topics from quantitative and qualitative research, by comparing current research traditions with comprehensive and rigorous judgements. Although "systematic reviews" are generally considered as rigorous, transparent, replicable, and unbiased ways to assess the quality of the evidence following a fixed process [77], they have considerable shortcomings as every other scientific method intrinsically acquire.

In summary, systematic reviews provide a more rigorous method of reviewing the literature to inform decision making, although the quality can be unreliable, and results are decontextualized. However, the purposes of this study were to focus on the risk and protective factors for the mental disorder, PTSD, and depression disorders, and secondly on the prevalence rates of the mentioned disorders, rather than the quality of the measures. A limitation of the present study is the fact that different sample populations in the selected studies made it difficult to conclude the exact prevalence rates. Refugees and asylum seekers that were studied in the current review had mostly Middle Eastern, African, or South Asian backgrounds. Although all the participating persons in selected studies were categorized as refugees or asylum seekers, they had different experiences of internal war in terms of the time, cultural, and religious backgrounds. One cannot sort all of them in a single category and generalize the findings to the whole refugee and asylumseeker population. We think that the latter limitation is very important as systematic reviews decontextualize the studies from their specific context.

\section{Conclusions}

The findings of the current study indicate that risks and protective factors for the mental health of forced migrants are multi-dimensional and closely knitted with the everyday lives of refugees and asylum seekers. To address the mental health risks and protective factors amongst vulnerable refugee populations, the application of the bottom-up approaches would be more feasible. There are clear potentials within refugee communities that if recognized by practice research and empowered by policy, sustainability of interventions and therapies would increase.

We highlighted the need for acquiring a holistic approach in the field of mental health of forced migrants and insisted that socio-ecological framework is a critical component for a sustainable and culturally relevant practice. When socio-ecological perspective is in the forefront of a social worker's approach to refugees, this vulnerable population's needs will be more sustainably served --in a more culturally responsive manner. In the context of the barriers refugees face in accessing mental health care, it serves at least in a rudimentary way as a useful framework in identifying, systematically organizing, and analyzing the determinants.

Author Contributions: Conceptualization, H.F., N.J., J.C.A., T.T. and M.T.; Methodology, H.F. and J.C.A.; Data extraction, H.F., N.J., and J.C.A.; Literature review, H.F. and N.J.; Supervision, J.C.A., T.T. and M.T. All authors have read and agreed to the current version of the manuscript.

Funding: This research received a grant from The Finnish National Agency for Education with a grant agreement number TM-18-11025. 
Conflicts of Interest: The authors declare no conflict of interest.

\section{References}

1. Siriwardhana, C.; Ali, S.S.; Roberts, B.; Stewart, R. A Systematic Review of Resilience and Mental Health Outcomes of Conflict-Driven Adult Forced Migrants. Conflict and health 2014, 8, 13.

2. Hall, B.J.; Olff, M. Global Mental Health: Trauma and Adversity among Populations in Transition. European journal of psychotraumatology 2016, 7, 31140-3.

3. Refugee Data Finder. 2020, 2021.

4. United Nations High Commissioner for Refugees. Global Trends: Forced Displacement in 2018. 2019.

5. Hynie, M. The Social Determinants of Refugee Mental Health in the Post-Migration Context: A Critical Review. The Canadian Journal of Psychiatry 2018, 63, 297-303.

6. Mölsä, M.; Tiilikainen, M.; Punamäki, R. Usage of Healthcare Services and Preference for Mental Healthcare among Older Somali Immigrants in Finland. Ethnicity \& health 2019, 24, 607-622.

7. Marmot, M. Health Equity in England: The Marmot Review 10 Years On. 2020.

8. Murray, K.E.; Davidson, G.R.; Schweitzer, R.D. Review of Refugee Mental Health Interventions Following Resettlement: Best Practices and Recommendations. American journal of orthopsychiatry 2010, 80, 576-585.

9. Hutchinson, M.; Dorsett, P. What does the Literature Say about Resilience in Refugee People? Implications for Practice. Journal of social inclusion 2012, 3, 55-78.

10. Kim Robinson. Voices from the Front Line: Social Work with Refugees and Asylum Seekers in Australia and the UK. The British journal of social work 2014, 44, 1602-1620.

11. Watters, C. Emerging Paradigms in the Mental Health Care of Refugees. Social science \& medicine (1982) 2001, 52, 17091718.

12. Li, S.; Liddell, B.; Nickerson, A. The Relationship between Post-Migration Stress and Psychological Disorders in Refugees and Asylum Seekers. Curr Psychiatry Rep 2016, 18, 1-9.

13. Cantor-Graae, E.; Selten, J. Schizophrenia and Migration: A Meta-Analysis and Review. The American journal of psychiatry 2005, 162, 12-24.

14. Lindert Jutta; Schinina Guglielmo. Mental health of refugees and asylum seekers. In Migration and Health in the European Union.; Rechel Bernd, Mladovsky Philipa, Deville Walter, Rijks Barbara, Petrova-Benedict Raumyana and Mckee Martin, Eds.; Open University Press: Berkshire, pp. 169-181.

15. Bogic, M.; Njoku, A.; Priebe, S. Long-Term Mental Health of War-Refugees: A Systematic Literature Review. BMC international health and human rights 2015, 15, 29.

16. Ottisova, L.; Smith, P.; Shetty, H.; Stahl, D.; Downs, J.; Oram, S. Psychological Consequences of Child Trafficking: An Historical Cohort Study of Trafficked Children in Contact with Secondary Mental Health Services. PloS one 2018, 13, e0192321.

17. Chen, W., PhD; Hall, B.J., PhD; Ling, L., Prof; Renzaho, A.M., Prof. Pre-Migration and Post-Migration Factors Associated with Mental Health in Humanitarian Migrants in Australia and the Moderation Effect of Post-Migration Stressors: Findings from the First Wave Data of the BNLA Cohort Study. The Lancet. Psychiatry 2017, 4, 218-229.

18. Hollander, A.; Dal, H.; Lewis, G.; Magnusson, C.; Kirkbride, J.B.; Dalman, C. Refugee Migration and Risk of Schizophrenia and Other Non-Affective Psychoses: Cohort Study of 1.3 Million People in Sweden. BMJ 2016, 352, i1030.

19. Howie, H.; Rijal, C.M.; Ressler, K.J. A Review of Epigenetic Contributions is î́p.ito Post-Traumatic Stress Disorder. Dialogues in clinical neuroscience 2019, 21, 417-428.

20. Moreno, A.; Piwowarczyk, L.; LaMorte, W.; Grodin, M. Characteristics and Utilization of Primary Care Services in a Torture Rehabilitation Center. J Immigrant Health 2006, 8, 163-171. 
21. Lamkaddem, M.; Stronks, K.; Devillé, W.D.; Olff, M.; Gerritsen, A.A.M.; Essink-Bot, M. Course of Post-Traumatic Stress Disorder and Health Care Utilisation among Resettled Refugees in the Netherlands. BMC psychiatry 2014, 14, 90.

22. Agrawal, P.; Venkatesh, A.K. Refugee Resettlement Patterns and State-Level Health Care Insurance Access in the United States. American journal of public health (1971) 2016, 106, 662-663.

23. de Anstiss, H.; Ziaian, T.; Procter, N.; Warland, J.; Baghurst, P. Help-Seeking for Mental Health Problems in Young Refugees: A Review of the Literature with Implications for Policy, Practice, and Research. Transcultural psychiatry 2009, 46, 584-607.

24. Colucci, E.; Minas, H.; Szwarc, J.; Guerra, C.; Paxton, G. In Or Out? Barriers and Facilitators to Refugee-Background Young People Accessing Mental Health Services. Transcultural psychiatry 2015, 52, 766-790.

25. Brown, M.; Glendenning, A.; Hoon, A.E.; John, A. Effectiveness of Web-Delivered Acceptance and Commitment Therapy in Relation to Mental Health and Well-being: A Systematic Review and Meta-Analysis. Journal of medical Internet research 2016, 18 , e221.

26. Rankopo, M.J.; Osei-Hwedie, K. Globalization and Culturally Relevant Social Work: African Perspectives on Indigenization. International social work 2011, 54, 137-147.

27. Kira, I.A.; Tummala-Narra, P. Psychotherapy with Refugees: Emerging Paradigm. Journal of loss \& trauma 2015, 20, 449467.

28. Bronfenbrenner, U. Contexts of Child Rearing: Problems and Prospects. The American psychologist 1979, 34, 844-850.

29. Kenneth R. McLeroy; Daniel Bibeau; Allan Steckler; Karen Glanz. An Ecological Perspective on Health Promotion Programs. Health education quarterly 1988, 15, 351-377.

30. Henderson, D.; Baffour, T. Applying a Socio-Ecological Framework to Thematic Analysis using a Statewide Assessment of Disproportionate Minority Contact in the United States. Qualitative report 2015, 20, 1960.

31. Kilanowski, J.F. Breadth of the Socio-Ecological Model. Journal of agromedicine 2017, 22, $295-297$.

32. Wong, W.C.W.; Cheung, S.; Miu, H.Y.H.; Chen, J.; Loper, K.A.; Holroyd, E. Mental Health of African Asylum-Seekers and Refugees in Hong Kong: Using the Social Determinants of Health Framework. BMC public health 2017, $17,153$.

33. Liberati, A.; Altman, D.G.; Tetzlaff, J.; Mulrow, C.; Gøtzsche, P.C.; Ioannidis, J.P.A.; Clarke, M.; Devereaux, P.J.; Kleijnen, J.; Moher, D. The PRISMA Statement for Reporting Systematic Reviews and Meta-Analyses of Studies that Evaluate Healthcare Interventions: Explanation and Elaboration. BMJ 2009, 339, b2700.

34. Alemi, Q.; James, S.; Montgomery, S. Contextualizing Afghan Refugee Views of Depression through Narratives of Trauma, Resettlement Stress, and Coping. Transcultural psychiatry 2016, 53, 630-653.

35. Page, M.J.; McKenzie, J.E.; Bossuyt, P.M.; Boutron, I.; Hoffmann, T.C.; Mulrow, C.D.; Shamseer, L.; Tetzlaff, J.M.; Akl, E.A.; Brennan, S.E. et al. The PRISMA 2020 Statement: An Updated Guideline for Reporting Systematic Reviews. Systematic reviews 2021, 10, 89 .

36. Affleck, W.; Thamotharampillai, U.; Jeyakumar, J.; Whitley, R. "If One does Not Fulfil His Duties, He must Not be a Man": Masculinity, Mental Health and Resilience Amongst Sri Lankan Tamil Refugee Men in Canada. Cult Med Psychiatry 2018, $42,840-861$.

37. Alemi, Q.; Weller, S.C.; Montgomery, S.; James, S. Afghan Refugee Explanatory Models of Depression: Exploring Core Cultural Beliefs and Gender Variations. Medical anthropology quarterly 2017, 31, 177-197.

38. Im, H.; Ferguson, A.; Hunter, M. Cultural Translation of Refugee Trauma: Cultural Idioms of Distress among Somali Refugees in Displacement. Transcultural psychiatry 2017, 54, 626-652.

39. Omar, Y.S.; Kuay, J.; Tuncer, C. 'Putting Your Feet in Gloves Designed for Hands': Horn of Africa Muslim Men Perspectives in Emotional Wellbeing and Access to Mental Health Services in Australia. International journal of culture and mental health 2017, 10, 376-388.

40. Savic, M.; Chur-Hansen, A.; Mahmood, M.A.; Moore, V.M. 'We Don't have to Go and See a Special Person to Solve this Problem': Trauma, Mental Health Beliefs and Processes for Addressing 'mental Health Issues' among Sudanese Refugees in Australia. International journal of social psychiatry 2016, 62, 76-83. 
41. Poudel-Tandukar, K.; Jacelon, C.S.; Chandler, G.E.; Gautam, B.; Palmer, P.H. Sociocultural Perceptions and Enablers to Seeking Mental Health Support among Bhutanese Refugees in Western Massachusetts. International quarterly of community health education 2019, 39, 135-145.

42. Yaser, A.; Slewa-Younan, S.; Smith, C.A.; Olson, R.E.; Guajardo, M.G.U.; Mond, J. Beliefs and Knowledge about PostTraumatic Stress Disorder Amongst Resettled Afghan Refugees in Australia. International journal of mental health systems 2016, $10,31$.

43. Yassin, N.; Yassin, N.; Taha, A.; Taha, A.; Ghantous, Z.; Ghantous, Z.; Atoui, M.; Atoui, M.; Forgione, F.; Forgione, F. Evaluating a Mental Health Program for Palestinian Refugees in Lebanon. J Immigrant Minority Health 2018, 20, $388-398$.

44. Yu, S.; Jang, J.; Noh, J.; Kwon, Y.D.; Park, H.; Woo, J. What is it to be Mentally Healthy from the North Korean Refugees' Perspective?: Qualitative Research on the Changes in Mental Health Awareness among the North Korean Refugees. Psychiatry investigation 2018, 15, 1019-1029.

45. Vitale, A.; Ryde, J. Promoting Male Refugees' Mental Health After they have been Granted Leave to Remain (Refugee Status). The international journal of mental health promotion 2016, 18, 106-125.

46. Poole, D.N.; Hedt-Gauthier, B.; Liao, S.; Raymond, N.A.; Bärnighausen, T. Major Depressive Disorder Prevalence and Risk Factors among Syrian Asylum Seekers in Greece. BMC public health 2018, 18, 908.

47. Chung, M.C.; AlQarni, N.; AlMazrouei, M.; Al Muhairi, S.; Shakra, M.; Mitchell, B.; Al Mazrouei, S.; Al Hashimi, S. Posttraumatic Stress Disorder and Psychiatric Co-Morbidity among Syrian Refugees of Different Ages: The Role of Trauma Centrality. Psychiatr. Q. 2018, 89, 909-921.

48. Hocking, D.; Sundram, S. Demoralisation Syndrome does Not Explain the Psychological Profile of Community-Based Asylum-Seekers. Comprehensive Psychiatry 2015, 63, 55-64.

49. Campbell, M.R.; Mann, K.D.; Moffatt, S.; Dave, M.; Pearce, M.S. Social Determinants of Emotional Well-being in New Refugees in the UK. Public Health 2018, 164, 72-81.

50. Şimşek, Z.; Yentur Doni, N.; Gül Hilali, N.; Yildirimkaya, G. A Community-Based Survey on Syrian Refugee Women's Health and its Predictors in Şanliurfa, Turkey. Women \& Health 2018, 58, 617-631.

51. Dietrich, H.; Al Ali, R.; Tagay, S.; Hebebrand, J.; Reissner, V. Screening for Posttraumatic Stress Disorder in Young Adult Refugees from Syria and Iraq. Comprehensive Psychiatry 2019, 90, 73-81.

52. Georgiadou, E.; Zbidat, A.; Schmitt, G.M.; Erim, Y. Prevalence of Mental Distress among Syrian Refugees with Residence Permission in Germany: A Registry-Based Study. Frontiers in psychiatry 2018, 9, 393.

53. Schweitzer, R.D.; Vromans, L.; Brough, M.; Asic-Kobe, M.; Correa-Velez, I.; Murray, K.; Lenette, C. Recently Resettled Refugee Women-at-Risk in Australia Evidence High Levels of Psychiatric Symptoms: Individual, Trauma and Post-Migration Factors Predict Outcomes. BMC medicine 2018, 16, 149.

54. Segal, S.; Khoury, V.; Salah, R.; Ghannam, J. Contributors to Screening Positive for Mental Illness in Lebanon's Shatila Palestinian Refugee Camp. The Journal of Nervous and Mental Disease 2018, 206, 46-51.

55. Grupp, F.; Moro, M.R.; Nater, U.M.; Skandrani, S.M.; Mewes, R. "It's that Route that Makes Us Sick": Exploring Lay Beliefs about Causes of Post-Traumatic Stress Disorder among Sub-Saharan African Asylum Seekers in Germany. Frontiers in psychiatry 2018, 9, 628 .

56. Kandemir, H.; Karataş, H.; Çeri, V.; Solmaz, F.; Kandemir, S.; Solmaz, A. Prevalence of War-Related Adverse Events, Depression and Anxiety among Syrian Refugee Children Settled in Turkey. Eur Child Adolesc Psychiatry 2018, 27, 15131517.

57. Shawyer, F.; Enticott, J.C.; Block, A.A.; Cheng, I.; Meadows, G.N. The Mental Health Status of Refugees and Asylum Seekers Attending a Refugee Health Clinic Including Comparisons with a Matched Sample of Australian-Born Residents. BMC psychiatry 2017, 17, 76.

58. Slewa-Younan, S.; Yaser, A.; Guajardo, M.G.U.; Mannan, H.; Smith, C.A.; Mond, J.M. The Mental Health and Help-Seeking Behavior of Resettled Afghan Refugees in Australia. International journal of mental health systems 2017, 11, 56. 
59. Tinghög, P.; Malm, A.; Arwidson, C.; Sigvardsdotter, E.; Lundin, A.; Saboonchi, F. Prevalence of Mental Ill Health, Traumas and Post Migration Stress among Refugees from Syria Resettled in Sweden After 2011: A Population-Based Survey. BMJ Open 2017, 7, e018899.

60. Lillee, A.; Thambiran, A.; Laugharne, J. Evaluating the Mental Health of Recently Arrived Refugee Adults in Western Australia. Journal of Public Mental Health 2015, 14, 56-68.

61. Leiler, A.; Bjärtå, A.; Ekdahl, J.; Wasteson, E. Mental Health and Quality of Life among Asylum Seekers and Refugees Living in Refugee Housing Facilities in Sweden. Social psychiatry and psychiatric epidemiology 2019, 54, 543-551.

62. Rizkalla, N.; Segal, S.P. Well-Being and Posttraumatic Growth among Syrian Refugees in Jordan. Journal of Traumatic Stress 2018, 31, 213-222.

63. Acarturk, C.; Cetinkaya, M.; Senay, I.; Gulen, B.; Aker, T.; Hinton, D. Prevalence and Predictors of Posttraumatic Stress and Depression Symptoms among Syrian Refugees in a Refugee Camp. J. Nerv. Ment. Dis. 2018, 206, 40-45.

64. Pandya, S. Spirituality for Mental Health and Well-being of Adult Refugees in Europe. J Immigrant Minority Health 2018, 20, 1396-1403.

65. Schünemann, H.; Oxman, A.; Brozek, J.; Glasziou, P.; Bossuyt, P.; Chang, S.; Muti, P.; Jaeschke, R.; Guyatt, G. GRADE: Assessing the Quality of Evidence for Diagnostic Recommendations. ACP J. Club 2009, 149, 2.

66. Guyatt, G.H.; Oxman, A.D.; Montori, V.; Vist, G.; Kunz, R.; Brozek, J.; Alonso-Coello, P.; Djulbegovic, B.; Atkins, D.; Falck-Ytter, Y. et al. GRADE Guidelines: 5. Rating the Quality of Evidence-publication Bias. Journal of clinical epidemiology 2011, 64, 1277-1282.

67. Balshem, H.; Helfand, M.; Schünemann, H.J.; Oxman, A.D.; Kunz, R.; Brozek, J.; Vist, G.E.; Falck-Ytter, Y.; Meerpohl, J.; Norris, S. et al. GRADE Guidelines: 3. Rating the Quality of Evidence. Journal of clinical epidemiology 2011, 64, 401-406.

68. Carswell, K.; Blackburn, P.; Barker, C. The Relationship between Trauma, Post-Migration Problems and the Psychological Well-being of Refugees and Asylum Seekers. International journal of social psychiatry 2011, 57, 107-119.

69. Beiser, M.; Hou, F. Language Acquisition, Unemployment and Depressive Disorder among Southeast Asian Refugees: A 10Year Study. Social science \& medicine (1982) 2001, 53, 1321-1334.

70. Jenny Phillimore; Lisa Goodson. Problem Or Opportunity? Asylum Seekers, Refugees, Employment and Social Exclusion in Deprived Urban Areas. Urban studies (Edinburgh, Scotland) 2006, 43, 1715-1736.

71. Szaflarski, M.; Bauldry, S. The Effects of Perceived Discrimination on Immigrant and Refugee Physical and Mental Health. In Immigration and Health.; Anonymous .; Emerald Publishing Limited: United States, 2019; 19, pp. 173-204.

72. Valtonen, K. Social Work and Migration : Immigrant and Refugee Settlement and Integration /. Social work and migration : 2008 .

73. Wood, T. Who is a Refugee in Africa? A Principled Framework for Interpreting and Applying Africa's Expanded Refugee Definition. International journal of refugee law 2019, 31, 290-320.

74. Nickerson, A.; Bryant, R.A.; Silove, D.; Steel, Z. A Critical Review of Psychological Treatments of Posttraumatic Stress Disorder in Refugees. Clinical psychology review 2011, 31, 399-417.

75. van Wyk, S.; van Wyk, S.; Schweitzer, R.; Schweitzer, R. A Systematic Review of Naturalistic Interventions in Refugee Populations. J Immigrant Minority Health 2014, 16, 968-977.

76. Turrini, G.; Purgato, M.; Ballette, F.; Nosè, M.; Ostuzzi, G.; Barbui, C. Common Mental Disorders in Asylum Seekers and Refugees: Umbrella Review of Prevalence and Intervention Studies. International journal of mental health systems 2017, 11, 51.

77. Mallett, R.; Hagen-Zanker, J.; Slater, R.; Duvendack, M. The Benefits and Challenges of using Systematic Reviews in International Development Research. Journal of development effectiveness 2012, 4, 445-455.

78. 